(IPViol) may be important structural determinants of vulnerability to HIV and sexually transmitted infections (STIs). This study characterised the type and frequency of CLViol and IPViol experienced by FSWs in southern India and examined the relationship between client violence and inconsistent condom use (ICU) with clients.

Methods Data were analysed from cross-sectional surveys of FSWs in three districts in Karnataka state (2007). ICU was defined as condom use frequency reported as 'never/sometimes/often' vs 'always', by repeat and occasional clients. CLViol and IPViol measures included having experienced physical violence in the past 6 months (ie, hurt, hit, kicked, punched, choked, burned) or sexual violence in the past year (ie, beaten or otherwise physically forced to have sexual intercourse).

Results Of our sample of 1245 FSWs, ICU was $13.0 \%$ with occasional and $20.2 \%$ with repeat clients. Overall, $13.1 \%$ of women reported physical violence from any perpetrator (CLViol: $5.5 \%$; IPViol: $4.6 \%$ ) and $9.7 \%$ reported sexual violence (CLViol: 5.2\%; IPViol: 4.4\%). In multivariable logistic regression analysis, the odds of ICU with occasional clients were significantly higher for women who had experienced physical CLViol (adjusted OR (AOR): $2.4,95 \%$ CI: $1.2 \%$ to $4.8 \%$ ) or sexual CLViol (AOR: $2.7,95 \%$ CI: $1.3 \%$ to $5.5 \%$ ). Similar results were found with repeat clients: AOR: $2.5,95 \% \mathrm{CI}: 1.4 \%$ to $4.6 \%$ and AOR $2.3,95 \%$ CI: $1.3 \%$ to $4.2 \%$, for physical and sexual violence respectively. A dose-response relationship between the number of times experiencing CLViol and increased ICU with both types of clients was also observed $(p<0.001)$ (Abstract O2-S6.02 figure 1). IPViol was not significantly associated with ICU with clients. Experiencing CLViol was not associated with experiencing IPViol $(p=0.321)$.

Discussion A strong independent relationship between experiencing client violence and inconsistent condom use with clients among FSWs in southern India was observed, highlighting FSWs' vulnerability to HIV/STI infection. Occupational violence against FSWs should be addressed within HIV/STI prevention programming. Structural-legal reforms to current sex work laws and safer-environment interventions should be developed to reduce violence and HIV/STI vulnerability among FSWs.

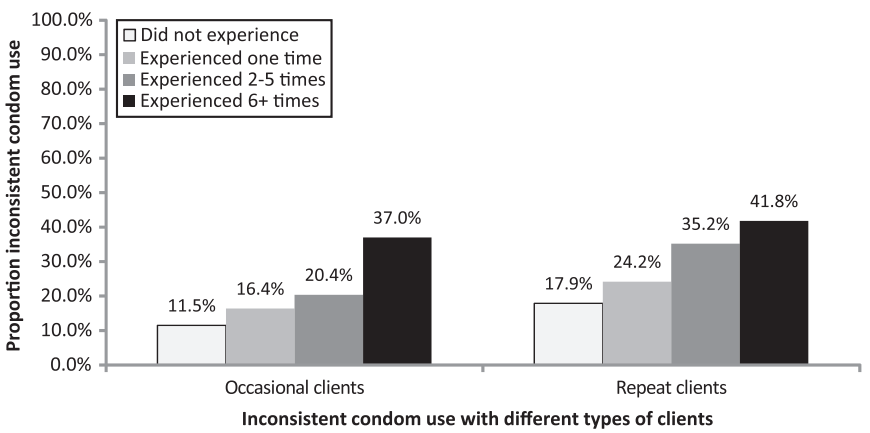

Abstract 02-S6.02 Figure 1 Proportion of female sex workers who used condoms inconsistently with occasional or repeat clients according to the number of times they experienced physical violence by clients in the last 6 months (did not experience, experienced one time, experienced 2-5 times, experienced $6+$ times).

\section{2-S6.03 HETEROGENEITY OF HIV RISK: FEMALE SEX WORKER CLIENTS AND THEIR NON-COMMERCIAL SEXUAL PARTNERS IN SOUTH INDIA}

doi:10.1136/sextrans-2011-050109.99

${ }^{1} \mathrm{~S}$ Shaw, ${ }^{2} \mathrm{~K}$ Deering, ${ }^{3} \mathrm{~S}$ Isac, ${ }^{3} \mathrm{~B}$ Ramesh, ${ }^{3} \mathrm{R}$ Washington, ${ }^{1} \mathrm{~S}$ Moses, ${ }^{1} \mathrm{~J}$ Blanchard. ${ }^{1}$ University of Manitoba, Winnipeg, Canada; ${ }^{2}$ University of British Columbia, Canada; ${ }^{3}$ Karnataka Health Promotion Trust, Bangalore, India

Introduction Clients of female sex workers (FSWs) are an important bridging population for the further transmission of HIV and other sexually transmitted infections (STIs). However, the extent of risk to non-commercial partners (NCP) of clients has rarely been quantified. This study sought to characterise the risk behaviours of clients with both non-commercial and commercial partners.

Methods Data were collected from a cross-sectional integrated behavioural and biological survey of FSW clients from five districts in Karnataka state, southern India. Clients were classified into three groups: married (and thus with an NCP); unmarried with at least one NCP; and unmarried without an NCP. Bivariate and multivariable logistic regression models were constructed to examine the association between group membership and condom use patterns with regular and casual FSWs, and where applicable, non-commercial partners. Associations between the prevalences of HIV, HSV-2, syphilis, gonorrhoeal and chlamydial infections and group membership were examined in multivariable models. Normalised weights were used to account for a complex sampling design.

Results The total sample size was 2328. Most respondents (61\%) were married, 9\% were unmarried with an NCP, and 30\% were unmarried without an NCP. Married clients were, on average 9 years older (34 vs 24.8 years), and had been visiting FSWs for an average of 12 years, vs 4 years in the other two marital categories. Compared to respondents without an NCP, married clients were at higher odds of reporting never using condoms with both casual (AOR: 1.8; 95\% CI: $1.3 \%$ to $2.4 \%, p<0.0001$ ) and regular (AOR:1.8; $95 \%$ CI $1.2 \%$ to $2.7 \%, p=0.009)$ FSWs. Among the two groups reporting an NCP, $91 \%$ of married respondents reported never using condoms with their NCP, compared to $62 \%$ of unmarried clients (AOR: $5.2 ; 95 \% \mathrm{CI}$ $3.3 \%$ to $8.1 \%, p<0.0001)$. HIV prevalence was $6 \%, 5 \%$ and $8 \%$ among those that were married, those unmarried without an NCP, and those unmarried with an NCP, respectively $(p=0.384)$. For the same groups, HSV-2 prevalence was $37 \%, 16 \%$ and $19 \%(p<0.0001)$. In adjusted analyses, married respondents remained at highest odds of being infected with HSV-2 (AOR: 1.5 ; 95\% CI $1.1 \%$ to $2.1 \%$, $\mathrm{p}=0.006$ ).

Conclusion Married respondents were least likely to use condoms with both commercial and non-commercial sexual partners, while also having the highest prevalence of HSV-2. These findings illustrate the high risk posed to both commercial and non-commercial partners of married clients of FSWs.

\section{2-S6.04 AN EXPLORATORY SURVEY OF MALE SEX WORKERS AND HIV RISK IN AN URBAN AREA OF SOUTHWEST CHINA}

doi:10.1136/sextrans-2011-050109.100

${ }^{1} \mathrm{~B}$ N Yu, ${ }^{2} \mathrm{X}$ A Wang, ${ }^{2} \mathrm{~F}$ Yu, ${ }^{3} \mathrm{~J}$ Wang, ${ }^{1} \mathrm{~J}$ Blanchard. ${ }^{1}$ University of Manitoba, Winnipeg, Canada; ${ }^{2}$ Chengdu Gay Care Organization, Chengdu, China; ${ }^{3}$ China Male Tongzhi Health Forum, Chengdu, China

Background Little knowledge is available on risk behaviours of HIV and basic demographic characteristics in male sex workers (MSW) in the population of men who have sex with men (MSM) in China. The objective of this study is to explore the risk to HIV/STI of MSW in southwest China through a convenience sample of an HIV surveillance survey of MSM

Method Those who self-reported had been paid for sex or "engaging in money exchange for sex" were identified as MSW or "Money Boys". Data of the past five cycles (2004-2008) of an HIV surveillance survey were included in this study. Descriptive statistics were employed to describe demographics, sex practices, and HIV prevalence in these male sex workers.

Results A total of 117 men self-identified as MSW in five consecutive cross-sectional surveys (2004 n=54, $2005 \mathrm{n}=39,2006 \mathrm{n}=45,2007$ $\mathrm{n}=34$, and $2008 \mathrm{n}=45$ ). The median age of participants ranged from 25 to 27 years. In the 6 months prior to the survey, the median number of male sex partners ranged from 4 to 11; the median of 
number of female sex partners ranged from 0 to 2 ; over $90 \%(89 \%-$ $98 \%$ ) of MSW reported anal sex and $26 \%$ of them engaged in group sex; but proportions who reported always using condoms for anal sex were less than $60 \%$ (ranged from $28 \%$ to $60 \%$ ). HIV screening positive rate was $9 \%$ in both 2007 and 2008 see Abstract O2-S6.04 table 1 . HIV positive rate is associated with the number of male sex partners and condom use frequency in both 2007 and 2008.

Conclusion MSW have been emerging in the sex trade industry in China. Behavioural risk factors and high prevalence of HIV in the MSW survey sample signalled a need for further understanding of this special population. Effective public health intervention strategy and programs are in urgent demand.

Abstract 02-S6.04 Table 1 Demographic characteristics of men who provided sex services for money in 2008 survey

\begin{tabular}{lr}
\hline Income US \$/month & \\
No income & $9 \%$ \\
$<\$ 145$ & $9 \%$ \\
$\$ 145-285$ & $29 \%$ \\
$\$ 285-430$ & $20 \%$ \\
$\$ 430-570$ & $13 \%$ \\
$>\$ 570$ & $20 \%$ \\
Education & \\
Primary education & $13 \%$ \\
Junior high & $13 \%$ \\
High school & $47 \%$ \\
University & $27 \%$ \\
1st sex partner & \\
Male & $53 \%$ \\
Female & $44 \%$ \\
Place for soliciting sex partners & \\
Bar, dance-hall, tea house, club & $31 \%$ \\
Bath-house, massage parlour & $7 \%$ \\
Public place: park, toilet etc. & $20 \%$ \\
Internet & $38 \%$ \\
Other & $4 \%$ \\
Sex orientation & \\
Gay & $40 \%$ \\
Heterosexual & $40 \%$ \\
Bi-sexual & $11 \%$ \\
Not sure & $40 \%$ \\
Last 6 months sex with men & $9 \%$ \\
Last 6 month sex with women & $98 \%$ \\
Group sex activity & $40 \%$ \\
\hline & $27 \%$ \\
\hline
\end{tabular}

\section{2-S6.05 SEXUAL VIOLENCE AGAINST MEN WHO HAVE SEX WITH MEN AND TRANSGENDERS (MSM-T) IN SOUTHERN INDIA}

doi:10.1136/sextrans-2011-050109.101

${ }^{1} \mathrm{~S}$ Shaw, ${ }^{1} \mathrm{R}$ Lorway, ${ }^{2} \mathrm{~K}$ Deering, ${ }^{3} \mathrm{H} \mathrm{L}$ Mohan, ${ }^{3} \mathrm{P}$ Bhattacharjee, ${ }^{3} \mathrm{~S}$ Isac, ${ }^{3} \mathrm{~B}$ M Ramesh, ${ }^{3} \mathrm{R}$ Washington, ${ }^{1} \mathrm{~S}$ Reza-Paul, ' $\mathrm{S}$ Moses, ${ }^{1} \mathrm{~J}$ Blanchard. ${ }^{1}$ University of Manitoba, Winnipeg, Canada; ${ }^{2}$ University of British Columbia, Canada; ${ }^{3}$ Karnataka Health Promotion Trust, Bangalore, India

Introduction Sexual violence (SV) has been associated globally with vulnerability to HIV and other sexually transmitted infections (STIs) in marginalised populations. There is little information on SV among men who have sex with men and transgendered individuals (MSM-T) in South Asia. Societal sanctions resulting in stigma and discrimination may place MSM-T at especially high risk for both SV and HIV/STIs. We investigated factors associated with SV among MSM-T, and the relationship between health care utilisation patterns and reported SV.
Methods Data were obtained from cross-sectional surveys in four districts in Karnataka state, south India. Bivariate and multivariable logistic regression models were constructed to examine factors related to SV. Multivariable negative binomial regression models examined the association between physician visits and SV Normalised weights were used to account for a complex sampling design.

Results The total sample size was 543 . The prevalence of SV in the past year was $18 \%$, with a range by district of $12 \%-31 \%$. HIV prevalence among those reporting SV was $20 \%$, compared to $12 \%$ among those not reporting SV. In a multivariable model, among those reporting sex work involvement, having anal sex with five or more casual sex partners in the past week was associated with SV (AOR: 4.1 ; $95 \% \mathrm{CI}$ : $1.2 \%$ to $14.3 \%, \mathrm{p}=0.03$ ). Of those not reporting sex work, feminised sexual identities (AOR: 6.10; 95\% CI: $1.3 \%$ to $28.8 \%, p=0.03$ ) and younger age (AOR: $0.9 ; 95 \% \mathrm{CI}: 0.9 \%$ to $1.0 \%$, $\mathrm{p}=0.002$ ) were associated with SV. There was no significant association between numbers of partners and SV among those not reporting sex work. Increased physician visits among those reporting SV was reported only for those involved in sex work (AOR: $1.7 ; 95 \% \mathrm{CI}: 1.1 \%$ to $2.7 \%, \mathrm{p}=0.01$ ).

Conclusions There was a trend towards higher HIV prevalence overall among MSM-T reporting sexual violence, as well higher HIV prevalence among important sub-groups experiencing SV. These findings highlight the importance of integrating programs to address sexual violence into STI/HIV prevention programs among MSM-T, and of understanding that there is considerable contextual heterogeneity in vulnerability to violence in this population. The demonstrated higher rates of physician utilisation by male sex workers who report sexual violence suggests an important point of contact for a population which may otherwise be quite hidden.

\section{2-S6.06 SEXUAL PRACTICES OF TRANSGENDER COMMUNITY IN PAKISTAN}

doi:10.1136/sextrans-2011-050109.102

\section{Z Ahmad. Al-Aser, Muzaffargarh, Pakistan}

Issue Pakistan, the second most populous Muslim nation in the world, has started to finally experience and confront the HIV/AIDS epidemic. The country had been relatively safe from any indigenous HIV cases for around two decades, with most of the infections being attributable to deported HIV positive migrants from the Gulf States. However, the virus finally seems to have found a home-base, as evidenced by the recent HIV outbreaks among the injection drug user community. Extremely high-risk behaviour has also been documented among Hijras (sex workers) and long-distance truck drivers. The weak government response coupled with the extremely distressing social demographics of this South-Asian republic also helps to compound the problem. The time is ripe now to prepare in advance, to take the appropriate measures to curtail further spread of the disease. If this opportunity is not utilised right now, little if at all could be done later. Objective: To assess risk behaviours including number and type of sex partners, condom use, knowledge of STIs and HIV/AIDS among hijras (eunuch) of Lahore, Pakistan.

Methods Two hundred hijras were recruited through Respondent Driven Sampling and interviewed by a team of experienced interviewers. Lessons Learnt: The mean age of the respondents was $29.2 \pm 6.3$ years (Range 18-55). More than two third (68.5\%) were illiterate; $23 \%$ were married. Among married, $89 \%$ were married to women and had 1 to 7 children. Sixty per cent had taken some hard drug (Cocaine, Heroin, Morphine and Amphetamine) during the last 12 months and 3\% had injected drugs. Eight per cent had sex with a woman during the last year. During the last 1 week, $82 \%$ respondents had 1-21 new clients and 69.5\% never used condom; $21.5 \%$ 